\title{
The representation of LGBTQ+ people in medical assessment and teaching scenarios
}

\author{
Authors: Emma Alexander, ${ }^{A}$ Ronan Lyne, ${ }^{B}$ Yousef Sasha Mottaghi-Taromssari ${ }^{B}$ and Isabel Lever ${ }^{B}$
}

\section{Introduction}

Examinations and teaching at UK medical schools are meant to be targeted towards common clinical scenarios faced by junior doctors in the NHS. This should include preparing students to provide equal treatment to groups with protected characteristics, including lesbian, gay, bisexual, transgender, queer+ (LGBTQ+) people. However, existing research has implied such groups face discrimination when receiving healthcare. ${ }^{1}$ This research project aims to:

> explore whether LGBTQ+ people are adequately represented in assessment and teaching scenarios

> explore whether medical students feel comfortable discussing sexuality in clinical scenarios

> explore whether prejudiced attitudes towards LGBTQ+ people are present in medical settings.

\section{Materials and methods}

Participants were current medical students at all stages of training. Participants completed a survey on Google Forms in mid-2018, distributed on public forums (year group pages, MSA Newsletter). The questions were developed both with a Likert scale (1-5, where $1=$ strongly disagree and $5=$ strongly agree) and with free text space where applicable. The data analysis used descriptive statistics on the questionnaire responses. This study received minimal risk clearance (Reference MRS-17/18-8128 and MRS-17/18-8129).

\section{Results and discussion}

A total of 107 medical students participated. The majority (81\%) attended one London-based medical school. The most common stage of training was in the final clinical year (38\%). Overall, $72 \%$ of students had been taught using educational scenarios including LGBTQ+ people, but fewer remembered LGBTQ + representation in OSCEs (21\%) and written exams (29\%). When asked whether $\mathrm{LGBTQ}+$ people were adequately represented in assessments and teaching scenarios, the median response was 2 (disagree). When asked whether $L G B T Q+$ people most commonly appear in scenarios when the diagnosis is related to sexuality or sexually transmitted diseases, the median response was 4 (agree). Medical students were generally comfortable with discussing sexual orientation (median response 4 (agree)). Participants generally felt medical school was an accepting environment for LGBTQ+ people (median response 4 (agree)) but felt less confident about the NHS working environment (median response 3 (unsure)).

\section{Conclusion}

Our study shows there is limited overt representation of LGBTQ+ people in medical school teaching and assessment scenarios. Current representation predominantly focuses on sex/STD-related scenarios, which may encourage bias in the assessment of LGBTQ+ patients. This finding therefore has implications not only for patient care but also for the satisfaction and comfort of LGBTQ+ individuals undergoing medical training. Further work should be done to update curricula and examinations to ensure adequate representation of $L G B T Q+$ people at all stages.

\section{Conflicts of interest}

None declared.

\section{Reference}

1 Elliott MN, Kanouse DE, Burkhart Q et al. Sexual minorities in England have poorer health and worse health care experiences: a national survey. J Gen Intern Med 2014;30:9-16.

Authors: APaediatric Liver, GI and Nutrition Centre and MowatLabs, London, UK; ' King's College London, London, UK 